\title{
Propriedades respiratórias do sangue e soluçōes de hemoglobina da piranha $\left(^{(*)}\right.$
}

\author{
Stephen C. Wood (') \\ Roy E. Weber $\left({ }^{2}\right)$ \\ Dennis A. Powers ( $\left.{ }^{3}\right)$
}

\section{Resumo}

As propriedades respiratórias do sangue da piranha, são diferentes das de outros peixes, prima riamente pela alta capacidade tamponante do $\mathrm{CO}_{2}$ $\left(\Delta \mathrm{HCO}_{-3} / \Delta \mathrm{pH}=19,6 \mathrm{mmol} / \mathrm{L}\right.$ para o sangue oxigenado e $39,1 \mathrm{mmol} / 1$ para o sangue desoxigenado). A concentração de nucleosídeos trifosfato (NTP) e a tensão de meia de saturação $\left(\mathrm{P}_{50}\right)$ do sangue total foi encontrado como sendo inversamente relacionado ao tamanho do corpo. O maior $\mathrm{P}_{50}$ em peixes pequenos, análogo aos valores obtidos em estudos prévios, envolvendo comparações inter-específicas, poderia ser uma adaptação ao mais alto índice metabólico específico ao peso. Tanto o ATP como a guanosina trifosfato (GTP), reduziram a afinidade pelo oxigênio das soluções de hemoglobina purificadas, considerando a correlação de tamanho dependente de $P_{50}$ e a concentração de NTP no sangue total. Embora em concentrações similares, nos eritrócitos, o GTP é mais potente que o ATP, como um modificador alostérico da funçäo da hemoglobina.

\section{INTRODUÇÃO}

A fisiologia respiratória da fauna aquática na Bacia Amazônica é interessante, por causa da grande diversidade de habitats e das propriedades respiratórias na água. O grande número de peixes de respiração aérea encontrado no Amazonas, demonstra a hipoxia predominante da água. Muita atenção tem sido dada à fisiologia respiratória destes peixes de respiração aérea facultativa e obrigatória (cf. Johansen, 1970), porém pouco é conhecido a respeito da função respiratória em espécies que devem sobreviver graças à sua respiração branquial. A piranha pareceu-nos particularmente interessante, pelo seu notável comportamento agressivo (presumivelmente, depen- dendo dos altos níveis do metabolismo aeróbico) e ubigüidade, sendo encontrada tanto em pântanos estagnados como em águas correntes.

Este trabalho descreve as propriedades respiratórias do sangue da piranha e da hemoglobina purificada e a modulação destas propriedades tamponantes do sangue, por fosfatos orgânicos. O transporte de oxigênio e as propriedades tamponantes do sangue, são comparadas com dados de outros peixes amazônicos (Powers et al., 1978). Também uma inesperada descoberta, a de que a afinidade pelo oxigênio e níveis de fosfato orgânico são dependentes do tamanho é discutida com respeito à velocidade metabólica dependente do tamanho.

\section{MATERIAIS E MÉTODOS}

As piranhas vermelhas (Serrasalmus rhombius) foram capturados com rede de arrasto ao longo das margens do rio Amazonas próximo a Manaus, Brasil. O sangue foi obtido por punçăo cardíaca, do peixe levemente anestesiado com sulfonato de trocaína metano (MS222, Sandoz).

Curvas de dissociação do oxigênio do sangue total foram determinadas a $30^{\circ} \mathrm{C}$, usando o Hem-o-Scan (American Inst. Co.) como descrito por Powers et al. (1978). O fator Bohr $\left(\Delta \log \mathrm{P}_{50} / \Delta \mathrm{pH}\right.$ foi obtido através das curvas de dissociação de oxigênio, obtidas em diferentes valores de $\mathrm{pH}$ (diferentes concentrações de $\mathrm{CO}_{2}$ ). A concentração do nucleosídeo trifosfato (NTP) nos eritrócitos, foi determinado por um método enzimático (Sigma Chemical CO., St. Louis). Ademais, as frações

$\left({ }^{\bullet}\right)$ - Versão original inglesa publicada em Comp. Biochem. Physiol. 62 A (1). 1979.

(1) - Department of Physiology, University of New Mexico, School of Medicine, Albuquerque, New Mexico 87131.

(2) - Departament of Zoophysiology. Aarhus University, DK 8000 Aarhus C. Denmark.

(3) - Department of Biology, Johns Hopkins University, Baltimore, Maryland 21218. 
derivado total de NTP da Adenosina trifosfato (ATP) e guanosina trifosfato (GTP), foram determinados usando cromatografia em camada delgada (Johansen et al., 1976) .

A capacidade tamponante do sangue definido como $\Delta \mathrm{HCO}_{3} / \Delta \mathrm{pH}$ (unidades = $\mathrm{mmol} / \mathrm{L} / \mathrm{pH}$ ), foi determinado a partir de medidas de $\mathrm{pH}$ com valores conhecidos de $\mathrm{PCO}_{2}$ usando um Radiômetro BMS-2 e bombas misturadoras de gás "Wosthoff". O bicarbonato foi calculado a partir da equação de HendersonHasselbalch $\left(\mathrm{HCO}_{3}^{-} \mathrm{mmol} / \mathrm{L}=10\left(\mathrm{pH}-\mathrm{pK}^{\prime}\right) . \propto\right.$. $\mathrm{PCO}_{2}$ ) usando $\mathrm{pK}^{\prime}$ e valores de solubilidade do $\mathrm{CO}_{2}(\propto)$ do plasma humano, a $30^{\circ} \mathrm{C}$ e o pH apropriado (Severinghaus, 1971) .

Os hemolisados foram preparados a partir de eritrócitos saturados de monóxido de carbono, lavados em solução salina que haviam sido congelados. A hemoglobina em solução foi quantitativamente reduzida pela adição de ditionito de sódio e diálise exaustiva contra $\mathrm{CO}_{2}$ saturado em tampão Tris $0,01 \mathrm{M}, \mathrm{pH} 7,5$ contendo $5 \times 10^{-4} \mathrm{M}$ de EDTA (Sullivan \& Riggs, 1967). A hemoglobina foi liberada dos ions orgânicos ou inorgânicos pela passagem através da coluna de troca iônica Mixed MB-3. Curvas de equilíbrio do oxigênio em soluções de hemoglobina foram medidas com o método de câmara de difusão do oxigênio (Niesel \& Thews, 1961) modificado como foi previamente descrito (Weber, Lykkeboe \& Johansen, 1976).

\section{RESUltados E DISCUSSÃo}

\section{PROPRIEDADES RESPIRATÓRIAS DO SANGUE}

Não há nada de extraordinário sobre a maioria das propriedades respiratórias do sangue da piranha. $O$ fator Bohr $\left(\Delta \log \mathrm{P}_{50} / \Delta \mathrm{pH}\right.$ $=-0,38$ ) e a afinidade pelo oxigênio (figura 1) são similares aos da maioria das outras espécies de respiração branquial no Amazonas (Powers et al., 1978). Do mesmo modo, o hematócrito $" \bar{x}=33,2 \%)$ e a concentração da hemoglobina ( $\bar{x}=1,24 \mathrm{mmol} / \mathrm{L}$ de sangue) são dignos de nota (tabela 1).

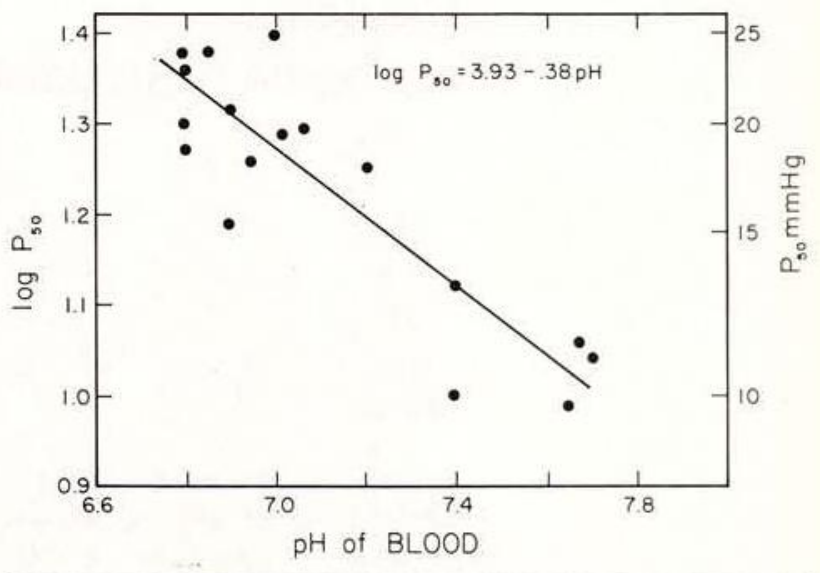

Fig. 1 - Dependência do $\mathrm{pH}$ na afinidade pelo oxigênio (medido como $\mathrm{P}_{50}$, pressão parcial de oxigênio $50 \%$ de saturação) do sangue total da piranha a $30^{\circ} \mathrm{C}$. A equação mostra os dados de regressăo dos minimos quadrados.

A capacidade tamponante do sangue da piranha é consideravelmente maior do que a da maioria dos peixes. Como é visto na (figura 2), a capacidade tamponante do sangue total $\left(\beta=\Delta \mathrm{HCO}^{-} / \Delta \mathrm{pH}\right)$ varia de 19,6 no sangue oxigenado até 39,1 no sangue desoxigenado. Em outro peixe não amazônico, examinado por Albers (1970), o valor de $\beta$ para o sangue total varia de 6,7 a 14,8 . As variações interes-

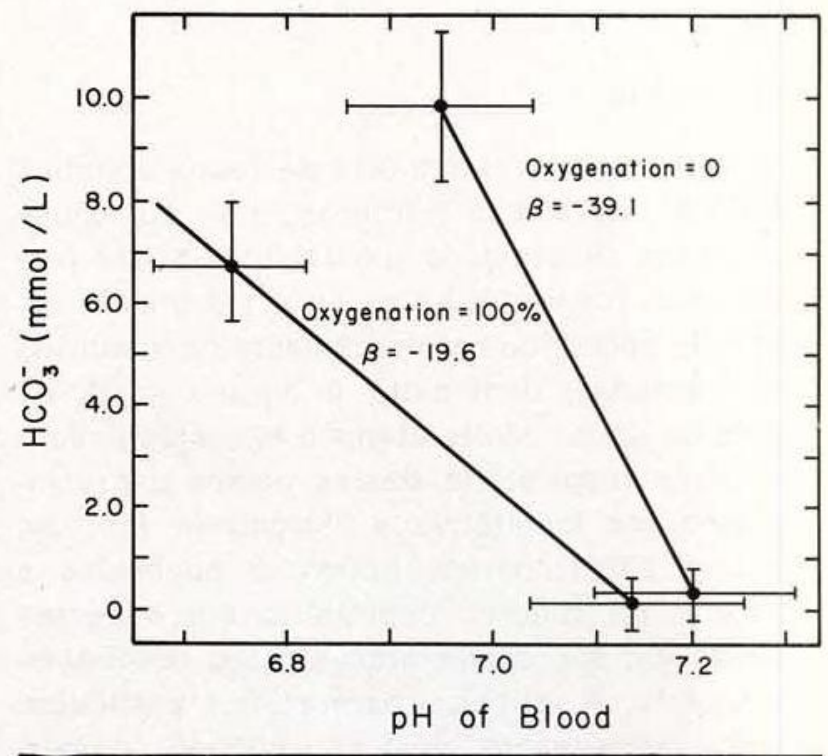

Fig. 2-Curvas de equilíbrio de $\mathrm{pH}, \mathrm{HCO}_{3}$ para o sangue total oxigenado e desoxigenado de Serrasalmus rhombeus $A 30^{\circ} \mathrm{C}$. Média \pm 2 vezes o Erro $\mathrm{Pa}$ drão da Média são indicados por barras verticaıs e horizontais. Beta $(\beta)$ é o grau de inclinação (capacidade tampão) em mmol $\mathrm{HCO}_{-} / \mathrm{L} / \mathrm{pH}$. 
TABELA 1. Niveis dos fosfatos orgânicos, tamanho corporal e afinidade pelo oxigênio no sangue total de Serrasalmus rhombeus.

\begin{tabular}{|c|c|c|c|c|c|c|}
\hline & $\begin{array}{l}\text { Peso } \\
\text { Corporal } \\
\mathrm{g}\end{array}$ & $\underset{\%}{\text { HCT }}$ & $\begin{array}{c}\text { mmoles de } \\
\text { Hemoglobin/L } \\
\text { de sangue }\end{array}$ & $\begin{array}{c}\text { mmoles de } \\
\text { NTP/L } \\
\text { de sangue }\end{array}$ & NTP/Hb & $\begin{array}{r}P_{50} \mathrm{mmHg} \\
\left(\mathrm{H}^{7,5} ; 30^{\circ} \mathrm{C}\right)\end{array}$ \\
\hline & 110 & 35 & 1,06 & 2,09 & 1,97 & \\
\hline & 110 & 31 & 0,65 & 2,28 & 3,49 & \\
\hline & 250 & 40 & 1,55 & 4,41 & 2,84 & 15,5 \\
\hline & 260 & 30 & 1,48 & 1,70 & 1,15 & \\
\hline & 300 & 34 & 1,41 & 2,89 & 2,06 & 14,0 \\
\hline & 325 & 35 & 1,36 & 2,45 & 1,80 & 13,6 \\
\hline & 350 & 30 & 1,08 & 1,42 & 1,32 & 11,0 \\
\hline & 400 & 30 & 1,28 & 1,24 & 0,97 & 10,0 \\
\hline & 600 & 34 & 1,29 & 1,75 & 1,36 & 11,2 \\
\hline $\bar{x}$ & 300,6 & 33,2 & 1,24 & 2,24 & 1,88 & 12,6 \\
\hline$\hat{D} . P$. & 149,7 & 3,3 & 0,27 & 0,96 & 0,83 & 2,1 \\
\hline
\end{tabular}

pecíficas das capacidades tamponantes são consideravelmente reduzidas, quando são corrigidas as diferenças na concentração de hemoglobina que é o maior tampão além do bicarbonato. Este valor da capacidade tamponante específica $\left(\Delta \mathrm{HCO}_{3} / \Delta \mathrm{pH} / \mathrm{Hb}\right)$, varia de 0,117 até $0,230 \mathrm{mmol} / \mathrm{L} / \mathrm{pH} / \mathrm{gHB}$ nas espécies examinadas por Albers (1970). Usando uma concentração de hemoglobina de 79,9 g/L $(1,24$ $\mathrm{mmol} / \mathrm{L} \times 64,456 \mathrm{~g} / \mathrm{mmol}$; tabela 1), a capacidade tamponante específica é de 0,24 e 0,49 para o sangue oxigenado e reduzido, esta é uma vantagem evidente para a piranha visto que a capacidade tampão é considerada um fator limitante para a atividade aeróbica no peixe (Hochachka, 1961). As curvas tampão mostram na figura 2, que estão deslocadas em baixo e para a esquerda dada a presença de ácido metabólico (presumivelmente lático) no sangue. Isto ocorre freqüentemente quando o peixe é manuseado e removido da água para retirar-se a amostra sangüínea. De qualquer modo, isto não altera o grau de inclinação $\beta$, das curvas tampão (cf. Siggaard-Andersen, 1974). Um resultado inesperado é aparentemente a grande dependência do $\mathrm{pH}$ do efeito de Haldane, resultando em um grande $\triangle \mathrm{HCO}^{-}$, com a oxigenação a baixos valores de $\mathrm{pH}$. Isto é diferente no sangue de mamíferos e de outros vertebrados onde dentro dos limites fisiológicos, as curvas para o sangue óxi e desóxi são paralelas. Os presentes resultados podem refletir uma forte dependência do $\mathrm{pH}$ na ligação de oxigênio e na união de carbamino.

\section{EFEITOS DO TAMANHO CORPORAL}

A descoberta de que no eritrócito o NTP e o $P_{50}$ estão inversamente relacionados ao peso do corpo não havia sido relatada anteriormente pelo menos que seja do nosso conhecimento. Embora o tamanho da amostra seja pequeno, a relação é bastante surpreendente (fig. 3). As variações do $P_{50}$ e do fosfato orgânico são opostas aos encontrados no desenvolvimento pós-natal dos mamímeros (cf. Dhindsa et al., 1972) e de outros vertebrados (cf. Manwell, 1960), mas a relação do $P_{s o}$ com o peso corporal é similar à observada, em comparações interespecificas (Schmidt-Nielsen e Larimer, 1958) .

O significado fisiológico deste fenômeno refere-se à relação alométrica entre o índice metabólico e o tamanho do corpo (cf. Hemmingsen, 1960). Comparações interespecíficas, revelam um aumento exponencial na demanda de oxigênio segundo o peso corporal nas espécies pequenas (cf. Schmidt-Nielson, 1975). Esta relação é dada pela equação :

$$
\mathrm{v}_{\mathrm{o}_{2}}=\mathrm{aM}^{\mathrm{b}}
$$


onde "a" é a intercepção na ordenada, "b" é a inclinação da reta expressa em logarítmos, " $M$ " é o peso corporal em gramas. A mesma relação tem sido demonstrada a um nível intraespecífico em "guppy" (Lebistes reticulatus) por Heusner et al. (1963). Para mamífe ros esta equação se torna :

$$
\mathrm{v}_{\mathrm{o}_{2}}=3,8 \mathrm{M}, 75
$$

(cf. Schmidt-Nielsen, 1975). Para peixes e outros poiquilotermos, o "custo de vida relativo", indicado pela intercepção "a", é cerca de 10 vezes menor (mesmo a $37^{\circ} \mathrm{C}$ ), mas a inclinação "b" é normalmente a mesma (cf. Brett, 1972). A $25^{\circ} \mathrm{C}$, a equação para um peixe "típico":

$$
\underset{2}{\mathrm{v}_{\mathrm{o}}} \simeq 0,75 \mathrm{M} \cdot 0,69
$$

(Hemmingsen, 1960). A importância desta relação alométrica é o aumento desigual na demanda de oxigênio por grama de peso corporal para os animais menores. Por exemplo, resolvendo a equação:

$$
\underset{2}{v_{0}} / M=0,75 M-0,31
$$

para um peixe hipótético pesando 10,000; 1,$000 ; 100,10$ e $1 \mathrm{~g}$, nos dá os seguintes valores de $\mathrm{V}_{02}\left(\mathrm{ml} \times \mathrm{g}^{-1} \times \mathrm{hr}^{-1}\right): 0,04 ; 0,09 ; 0,18$; 0,$37 ; 0,75$. Este efeito experimental do aumento corporal claramente se opõe à capacidade de transporte de $\mathrm{O}_{2}$ em peixes menores que $1000 \mathrm{~g}$.

Os corolários de que a mais alta demanda de oxigênio de peso específico em espécies menores é aumentada pela densidade capilar (Schmidt-Nielsen \& Pennycuik, 1961) e reduzem a afinidade pelo oxigênio do sangue (Schmidt-Nielsen \& Larimer, 1958). O último é considerado adaptativo, pois proporciona uma maior liberação de $\mathrm{O}_{2}$ para os tecidos, suprindo-os de $\mathrm{O}_{2}$ em uma alta pressão parcial. Impõe-se considerar que a reduzida afinidade pelo oxigênio do sangue em piranhas pequenas é adaptativa a um aumento de necessidade de $\mathrm{O}_{2}$. Este argumento foi proposto anteriormente em comparações interespecíficas em mamíferos (Schmidt-Nielsen \& Larimer, 1958),

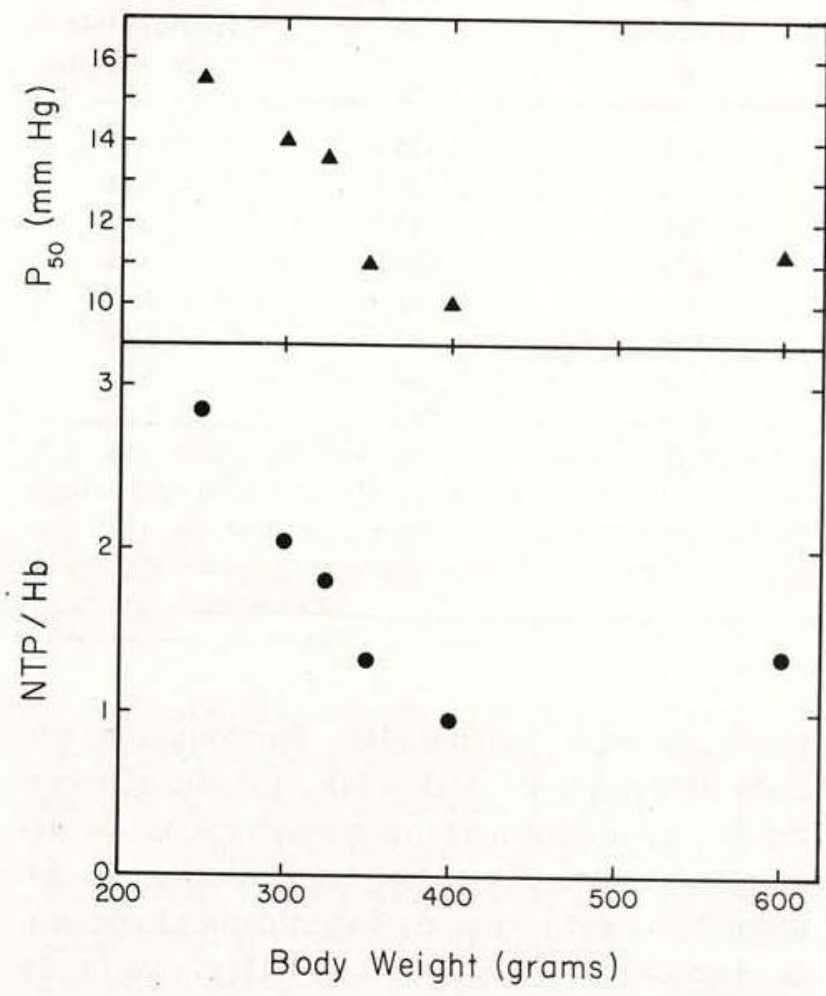

Fig. 3-Relação entre o tamanho corporal e afinidade pelo oxigênio (quadro superior) e proporção de fosfato orgânico/hemoglobina (quadro inferior) em Serrasalmus rhombeus. Os valores de $P_{50}$ são a pH 7.5 e $30^{\circ} \mathrm{C}$. NTP (nucleosídeo trifosfato).

répteis (Bennett, 1973) e pássaros (Lutz et al., 1974) onde $P_{50}$ aumenta com a diminuição do peso corporal. Desde que a saturação arterial seja mantida na porção superior horizontal da curva de dissociação, uma curva deslocada para a direita resulta na liberação de uma dada quantidade de oxigênio em altos valores de $\mathrm{PO}_{2}$ do tecido, o qual por sua vez, possibilita um aumento de velocidade de difusão de $\mathrm{O}_{2} \mathrm{e}$ índice metabólico do tecido. O presente trabalho mostra que a consideraçâo acima pode também ser aplicada a uma espécie, i.e., que indivíduos pequenos têm sangue com uma baixa afinidade pelo $\mathrm{O}_{2}$. Também é de interesse que a baixa afinidade pelo $\mathrm{O}_{2}$, resulta de diferenças relacionadas ao tamanho em eritrócitos com concentração de fosfato orgânico (veja abaixo) . 
PROPRIEDADES DE SOLUÇÕES DE HEMOGLOBINA

Experimentos de cromatografia em camada delgada mostram que eritrócitos contêm quantidades significantes, tanto de GTP como de ATP. Em quatro espécimes, os valores de ATP e GTP (média \pm desvio padrão) foram $0,66 \pm$ 0,09 e $0,62 \pm 0,26 \mathrm{mmol} / \mathrm{L}$ de sangue, respectivamente.

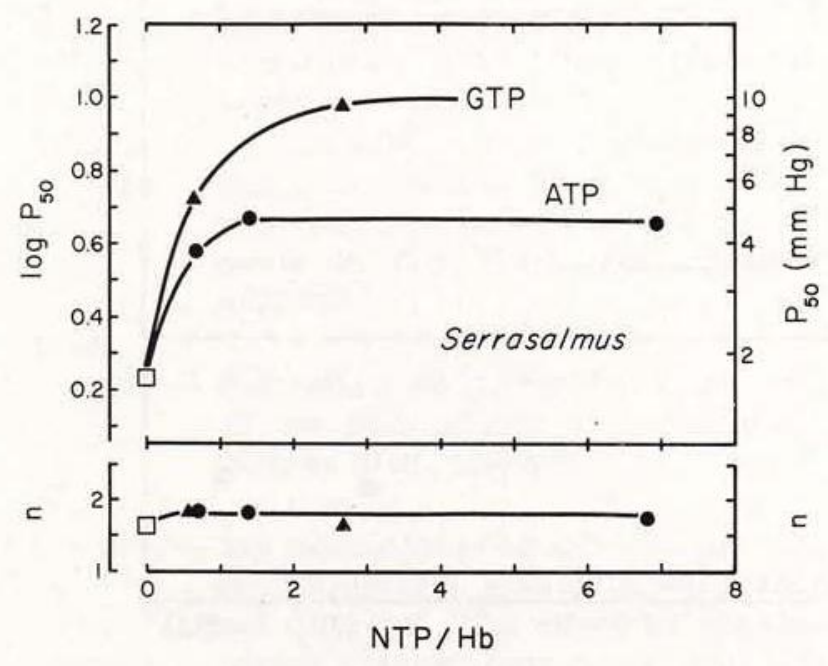

Fig. 4 - Influência do $\mathrm{pH}$ na afinidade pelo oxigênio $\left(P_{50}\right)$ e o coeficiente de Hill (n) a $20^{\circ} \mathrm{C}(\mathbf{A})$ e $30^{\circ} \mathrm{C}$ (-) da solução de hemoglobina fracionada de S. rhombeus. Tampão Tris, força iônica $=0,05$; concentração da hemoglobina $=0,62 \mathrm{mM}$ (tetrâmero)

Os valores de $P_{50}$ e " $n$ " (o coeficiente de Hill, indicando o grau de interação heme-heme) da hemoglobina fracionada de piranha e suas dependências de $\mathrm{pH}$ a $20^{\circ} \mathrm{C}$ e $30^{\circ} \mathrm{C}$ são mostradas na figura 4 . Na ausência de cofatores a hemoglobina tem uma alta afinidade pelo oxigênio (o $P_{50}$ é aproximadamente $1,6 \mathrm{~mm} \mathrm{Hg}$ e $\mathrm{pH} 7,3$ e $30^{\circ} \mathrm{C}$, comparado o valor correspondente de $14 \mathrm{~mm} \mathrm{Hg}$ no sangue total. O fator Bohr de soluções de hemoglobina é notavelmente dependente de $\mathrm{pH}$, mudando de valores aproximados de $\mathrm{O}_{2} \mathrm{pH} 7,3$ até cerca de $-1,0$ a valores de $\mathrm{pH}$ abaixo de 7,0 (veja abaixo).

$A$ grande diferença entre os valores de $P_{50}$ da solução de hemoglobina e do sangue, pode ser atribuída à presença de fosfatos orgânicos nos eritrócitos. Outros fatores que poderão contribuir para esta diferença, incluem uma alta concentração de hemo. blina nas células, a presença de $\mathrm{CO}_{2}$ no sangue, e o fato de que o $\mathrm{pH}$ intracelular de eritrócitos é mais baixa do que o $\mathrm{pH}$ plasmático, valor médido para o sangue total. Os últimos fatores podem também ser considerados pelo fato de que no $\mathrm{p}$ Habaixo de 7,0, o fator Bohr da solução de hemoglobina excede da do sangue total. Embora os fosfatos intensifi. quem o fator Bohr, na solução de hemoglobina (Benesch, Benesch Yu, 1969), dado o aumento das ligações a pH baixo, o efeito específico de $\mathrm{CO}_{2}$ no $\mathrm{P}_{5 \mathrm{c}}$ aumentando com o pH (Duhm, 1976) o qual contrabalança o efeito de fosfatos na sensibilidade do $\mathrm{pH}$.

O valor do calor aparente da oxigenação $(\Delta \mathrm{H})$ da hemoglobina é cerca de $-16,7 \mathrm{Kcal} /$ $\mathrm{mol}$ a $\mathrm{pH} 7,3$ para a variação da temperatura entre $20^{\circ}$ e $30^{\circ} \mathrm{C}$. Este valor inclui o calor de solução do oxigênio, e é similar aos valores encontrados para a maioria das hemoglobinas. O efeito da temperatura diminui em valores de $\mathrm{pH}$ mais baixos (figura 4) onde o efeito Bohr se torna operante, refletindo contribuições para o calor total de reaçẩo proveniente da oxigenação dos grupos ácidos na molécula.

Os efeitos dos nucleosídeos trifosfatos, ATP e GTP, na hemoglobina fracionada são mostrados na figura 5 . Ambos os fosfatos manifestam um grande efeito no $P_{50}$ em baixas proporções de fosfato/ hemoglobina. Este dado é consistente, com a observação de que variações na proporção de NTP/ hemoglobina no sangue total é de 0,8 a 2 e correlaciona-se com mudancas significativas no $P_{50}$ (figura 1 ). A figura 4 também mostra que, como em outras hemoglobinas de peixes investigadas, o GTP diminui a afinidade pelo oxigênio mais fortemente do que o ATP na mesma concentração. Isto mostra que, não obstante concentrações similares nos eritrócitos, o GTP poderá desempenhar um papel mais importante que o ATP, modificando a afinidade pelo oxigênio do sangue total. 


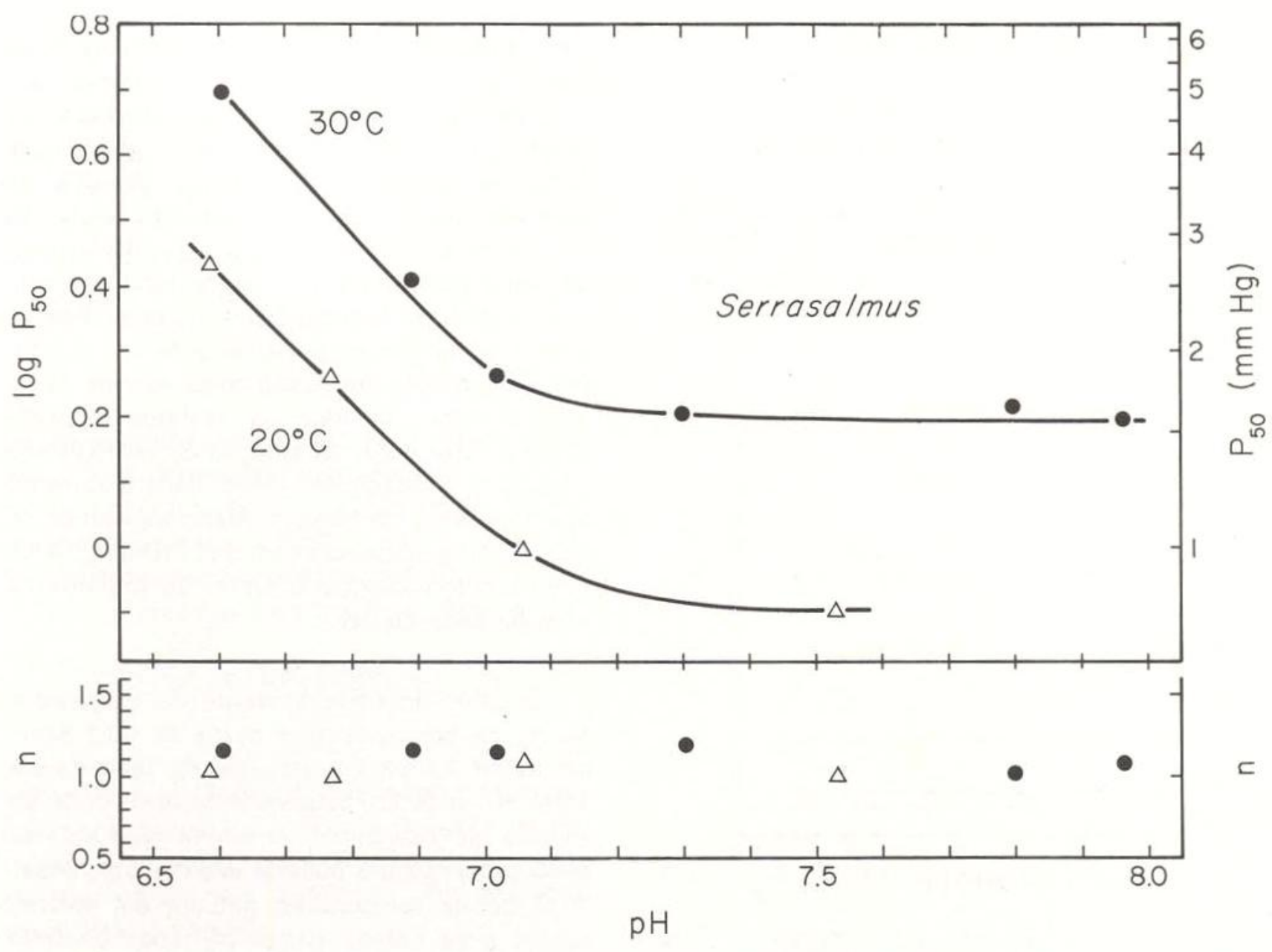

Fig. 5 - Efeitos do GTP e ATP na afinidade pelo oxigênio $\left(P_{50}\right)$ e o coeficiente de Hill (n) da hemoglobina fracionada de S. rhombeus. Tampão Tris, pH 7,30 força iônica $=0,05$, concentração da hemoglobina $=0,41$ mM (tetrâmero), $30^{\circ} \mathrm{C}$.

\section{Agradecimentos}

Esta pesquisa foi patrocinada pelo National Science Foundation, auxílio PCM 75-06451, o Danish Natural Science Council (R.E.W.) e National Institute of Health, auxílio HL-18026 (S.C.W.). Auxílio adicional foi fornecido pela N.S.F., auxílio DEB 76-19877 (D.A.P.) e pela National Geographic Society.

\section{SUMMARY}

Respiratory properties of piranha blood are distinguished from those of other fish primarily by the high $\mathrm{CO}_{2}$ buffering capacity $\left(\Delta \mathrm{HCO}_{3}^{-/} \mathrm{AH}=\right.$ $18.6 \mathrm{mmol} / \mathrm{L}$ for oxygenated blood and $39.1 \mathrm{mmol} / \mathrm{L}$ for deoxygenated blood). The concentration of nucleoside triphosphates (NTP) and the half-satu- ration tension $\left(P_{50}\right)$ of whole blood were found to be inversely related to body size. The higher $\mathrm{P}_{50}$ in smaller fish, analogous to values obtained in previous studies involving interspecies comparisons, could be adaptive to higher weight-specific metabolic rate. Both ATP and guanosine triphosphate (GTP) lowered the oxygen affinity of purified hemoglobin solutions, accounting for the size-dependent correlation of $\mathrm{P}_{50}$ and NTP concentration in whole blood. While similar in concentration in red cells, GTP is more potent than ATP as an allosteric modifier of hemoglobin function.

\section{BIBLIOGRAFIA}

Albers, $\mathrm{C}$.

1970 - Acid-base balance. In: Fish Physiology. (ed. HOAR, W.S. and RANDALL, D.J.) New York, Academic Press, 4:173-208. 
BENESCH, R.E.; BENESCH, R. \& YU, C.I.

1969 - The oxygenation of hemoglobin in the presence of 2, 3-diphosphoglycerate. Effect of temperature, $\mathrm{pH}$, ionic strength, and hemoglobin concentration. Biochemistry, 8:2567-2571.

BENNET, A.F.

1973 - Blood physiology and oxygen transport during activity in two lizards, Varanus gouldii and Sauromalus hispidus. Comp. Biochem. Physiol., 46a:673-690.

BRETT, J.R.

1972 - The metabolic demand for oxygen in fish, particularly salmonids, and a comparison with other vertebrates. Respir. Physiol. 14:151-170.

Dhindsa, D.A.; Hoverland, A.S. \& Templeton, J.W.

1972 - Postnatal changes in oxygen affinity and concentration of 2, 3-diphosphoglycerate in dog blood. Biol. Neonate, 20:226-235

Duнм, J.

1976 - Dual effect of 2, 3-diphosphoglycerate on the Bohr effects of human blood. Pflugers Arch., 263:55-60.

GILLEN, R.G. \& RigGs, A.

1971 - The hemoglobins of a fresh-water teleost, Cichlasoma cyanoguttatum (Baird and Girard) I. The effects of phosphorylated organic compounds upon the oxygen equilibria. Comp. Biochem. Physiol., 38b:585-595.

Hemmingsen, A.M.

1960 - Energy metabolism as related to body size and respiratory surfaces and its evolution. Rep. Steno. Mem. Hosp. Nord. Insulin Lab., 9:7-110.

Heusner, A.; KAyser, C.; MARK, C.; Stussi, T. \& HARMELIN, M.L.

1963 - Relation entre le poids et la consommation d'oxygene. Etude intraspecifique chez le poisson. C. R. Soc. Biol., 157: 654-657.

НоснАснКА, P.W.

1961 - Effect of physical training on oxygen debt and glycogen reserves in trout. Can. J. Zool., 39:767-776.

JOHANSEN, $\mathrm{K}$.

1970 - Airbreathing in fishes. In: Fish Physiology, 532. (ed. HOAR, W.S. \& RANDALL, D.J.). New York, Academic Press.

Johansen, K.; LyKKeboe, G.; Weber, R.E. \& Maloty, G.M.O.

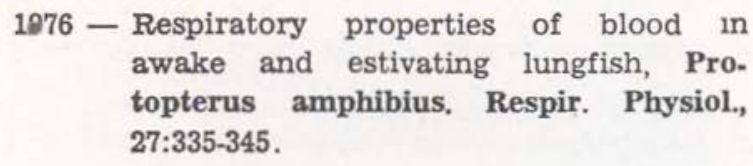
topterus amphibius. Respir. Physiol., $27: 335-345$
LUtz, P.L.; LONGMUiR, I.S. \& SChMidt-Nielsen, K. 1974 - Oxygen affinity of bird blood. Respir. Physiol., 20:325-330.

MANWELL, C.

1960 - Comparative physiology; blood pigments. Ann. Rev. Physiol., 22:191-244.

Niesel, W. \& Thews, G.

1961 - Ein neues verfahren zur dchnellen und genauen aufnahme der sauerstoffbindungskurve des blutes und konzen. trierter hamoproteidlosningen. Pflugers Arch. ges. Physiol., 273:380-395

POWers, D.A.; FYhN, H.J.; FYhn, E.U.H.: MARTiN, J.P.; Garlick, R.L. \& Wood, S.C.

1978 - Estudo comparativo de equilíbrio de oxigênio no sangue de 40 gêneros de peixes da Amazônia. Acta Amazonica 8(4) : Suplemento. (Este volume).

SCHMIDT-NIELSEN, K.

1975 - Animal physiology: adaptation and environment. Cambridge, Cambridge Univ. Press, p. 237.

SCHMIDT-NIELSEN, K. \& LARIMER, J.L.

1958 - Oxygen dissociation curves of mammalian blood in relation to body size. Amer. J. Physiol., 195:424-428

Schmidt-Nielsen, K. \& Pennycuik, P.

1961 - Capillary density in mammals in relation to body size and oxygen consumption. Amer. J. Physiol., 200:746-750.

SEVERINGHAUS, J.W

1971 - Carbon dioxide solubility and first dissociation constant ( $\mathrm{pK}$ ') of carbonic acid in plasma and cerebrospinal fluid: man. (ed. ALTMAN, P.L. \& DITTMER, D.S.), Bethesda. In: Handbook of Respiration and Circulation. Fed. Am. Soc. Exp. Biol., p. 218-219.

SiCK, H. \& GERSONDE, $\mathrm{K}$.

1969 - Method for registration of $\mathrm{O}_{2}$-binding curves of hemoproteins by means of a diffusion chamber. Analyt. Biochem., 32:362-376.

SIGGARD-ANDERSEN, O

1974 - The acid-base status of blood. Copenhagen, Munksgaard, p. 46.

Sullivan, B. \& Riggs, A.

1967 - Structure, function and evolution of turtle hemoglobins. II. Electrophoretic studies. Comp. Biochem. Physiol., 23: 449-458. 
W'eber, R.E.; LykKeboe, G. \& Johansen, K.

1976 - Physiological properties of eel hemoglobin: hypoxic acclimation, phosphate effects and multiplicity. J. Exp. Biol., 64:75-88.

WeBER, R.E.; WNOD, S.C. \& DAVIS, B.J.

1978 - Efeitos da respiração aérea sobre o balanço ácido-base no cascudo Hypostomus sp. Acta Amazonica 8(4) : Suplemento. (Este volume).
WOOD, S.C. \& JohaNSEN, K.

1972 - Adaptation to hypoxia by increased $\mathrm{HbO}_{2}$ affinity ann decreased red cell concentration. Nature New Biology, 237:278-279.

WOOD, S.C.; Johansen, K. \& Weber, R.E.;

1976 - Effects of ambient $\mathrm{PO}_{2}$ on red cell ATP and $\mathrm{HbO}_{2}$ affinity in the bottam-dwelling fish, Pleuronectes platessa. Respir, Physiol., 25:259-267. 